\title{
Platooning Control Using Visible Light Communications: A Feasibility Study
}

\author{
Mohammad Y. Abualhoul, Mohamed Marouf, Oyunchimeg Shagdar, and Fawzi Nashashibi
}

\begin{abstract}
The major benefits of driving vehicles in controlled close formations such as platoons are that of increasing traffic fluidity and reducing air pollution. While V2V communications is requisite for platooning stability, the existing radio communications technologies (e.g., the IEEE 802.11p) suffer from poor performance in highly dense road scenarios, which are exactly to be created by platooning. This paper studies the applicability of visible light communications (VLC) system for information exchange between the platoon members. A complete VLC model is built enabling precise calculations of Bit-Error-Rate (BER) affected by inter-vehicle distance, background noise, incidence angle and receiver electrical bandwidth. Based on our analytical model, the optical parameters suiting platooning application are defined. Finally, a SIMULINK model is developed to study the performances of a platooning longitudinal and lateral control, where VLC is used for vehicle-to-vehicle information exchange. Our study demonstrates the feasibility of VLC-based platooning control even in the presence of optical noise at significant levels and up to certain degree of road curvature.
\end{abstract}

\section{INTRODUCTION}

Nowadays, the requirements for the solution of road traffic problems such as accidents, roads congestion and the accompanying environmental pollution have exponentially increased. Both ordinary roads and highways are becoming more jammed every year due to inadequate road development to accommodate the growing number of vehicles. Automatically controlled vehicles in platoon whose inter-vehicle distance can be reduced down to 2 meters, will efficiently reduce the traffic jam by increasing the roads throughput. The data flow between vehicles is crucial to deliver information concerning the vehicle state (speed, acceleration, vehicle failure, brake, etc), for many road safety applications. For platooning control, V2V communication is needed to guarantee the string stability of the platoon [1].

Radio Frequency (RF) communication deploying IEEE $802.11 \mathrm{p}$ standard have been considered to facilitate relatively long range and high data rate communication for vehicular applications. However, the technology suffers from serious drawbacks, especially it may not always ensure stable communication due to increased channel congestion in highly dense vehicular traffic scenarios [2], [3], [4], indicating that the vehicular communication technologies still remains as an open problem.

Motivated by this, we consider a use of the vehicles' lightning system for inter-vehicle communications providing

*This work was not supported by any organization

The authors are with the IMARA research team, INRIA, Paris-Rocquencourt, FRANCE, e-mail: \{mohammad.abu_alhoul, mohamed.marouf, oyunchimeg.shagdar, fawzi.nashashibi $\} @$ inria.fr.

Manuscript is created on January 4, 2013; revised April 4, 2013. stable platooning. Compared to the radio communications technologies, VLC has a short history: it has been just standardized in 2011 [5] and its usage is considered for mainly indoor applications [6]. Nevertheless, its applicability for ITS outdoor usage has to be studied due to its extremely attractive features, including license-free wireless spectrum, low cost, dual functionality as lightning and communication source and its ability to take tremendous amount of load from RF channels.

Although, VLC for outdoor usage has been investigated for specific application such as traffic light to vehicle or infrastructures to train applications [7], there is no study made on the usage for platooning. We believe this paper is the first effort, which study its feasibility to vehicle platooning based on a complete analytical modeling.

In this paper, we develop a complete VLC channel and noise model taking account of background noise, incidence angle and receiver electrical bandwidth. collectivity and considering OOK (On-off Keying) modulation method, we calculated BER for varying inter-vehicle distance. Finally, using a SIMULINK, we simulate the impact of VLC on a platooning performance controlled under longitudinal and lateral control. Our simulation results show that it is feasible to achieve up to 7 meters Line-of-Sight (LOS) communication range even in the presence of optical noise at significant levels and with up to 60 degree of road curvature.

The remainder of the paper is organized as follows. After a brief review of the related work in Section II, a complete VLC channel model combined with longitudinal and lateral control model are presented in Section III. Section IV is dedicated to the performance evaluation and analytical BER simulation results of the VLC channel, together with different curvatures simulation results obtained from the platoon SIMULINK model. Finally, we conclude the paper in Section V.

\section{RELATED WORKS}

Since the 70's, platooning has been studied to increase the throughput of roads. PATH in California [8] and PRAXITELE in France [9] were the first pioneering projects. Later on, Auto21 CDS [10] focused on the smooth merging and splitting of platoon considering only highways for platooning-enabled cars only. In SARTRE project [11], platoons are considered fully autonomous with a common specially skilled driver, while all other vehicles are free to join and leave the platoon. A model of platooning vehicles with a constant inter-vehicle spacing has been presented in [12]. In this model, the vehicles communicate 
by broadcasting the event-driven type of messages through Dedicated Short Range Communications (DSRC), whereas periodic vehicle control-based messages use the infra-red (IR) spectrum in unicast. While IR is a popular media for LOS communications, compared to VLC, transmitting and receiving data in the IR spectrum is relatively high cost and requires specialized hardware. Our work differs from the previous research in the way that we focus on studying the feasibility of deploying the vehicles commercial lightning system in establishing communication link between platoon members.

There is a large body of literature investigating VLC links. Number of organizations, corporations and universities investigated and did researches on VLC technology. As an example and not exhaustively, Japan Electronics and Information Technology Industries Association's (JEITA) established standards for a Visible Light ID system in 2007. In 2008, specification standard were introduced by VLCC [13].

Experimental studies on VLC for outdoor applications are made in [14] and [7]. Using network simulator ns2, Liu [14] examined the key elements in realizing VLC networks based on experimental results and considering the constraints imposed by outdoor environment. Liu shows that VLC can satisfy the stringent reachability requirements for InterVehicle Communication (IVC) in dense vehicle traffic conditions. Where Cailean et al. [7] studied the both cooperation application between vehicles and road infrastructures using VLC and based on pure experimental demonstration, the authors presented a prototype of vehicular system proposing a cooperation between vehicles and road infrastructures, aiming to enhance traffic security. The main contributions of the above mentioned works are experimental and simulation study of VLC but not analytical study.

Some efforts have also been made on analytical modeling of VLC channels. Cailean in [7] provides day noise analysis together with VLC channel modeling adapting its functionalities to general outdoor applications. Lee in [15] presented an enhancement of out door VLC systems using selective combining receiver, while Okada in [16] proposed a roadto-vehicle VLC system using Light Emitting Diode (LED) traffic lights as transmitter and mainly discussed the receiver design challenges and noise effects. To our best knowledge, there are no published research papers concerning the analysis of VLC for platoon IVC environment.

\section{Platooning Control Using Visible Light COMMUNiCATIONS}

\section{A. VLC modeling}

The physical model of the optical communication system is shown in Fig. 1. The model shows half-duplex link between two vehicles in the platooning queue. Using this model, we relate to one hop, from the front vehicle to the rear vehicle. For multi-hop full duplex model, adding each hop to the last car and adding another connection between each two vehicles with reversal direction, may expand the single-hop physical model.

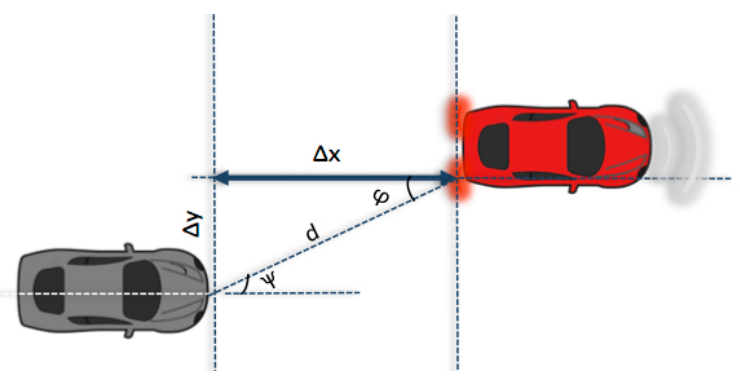

Fig. 1. Platoon optical communication system for V2V using rare light model. Where $\psi$ and $\varphi$ represents the both incidence and irradiance angles, $\mathrm{d}$ is the LOS direct inter-vehicle-distance.

Continual LOS between the rear LED and the receiver for channel modeling analysis is assumed, indicating that the platooned vehicles are free from any obstruction.

1) Channel DC Gain model: The VLC channel between each two vehicles in the platooning queue can be modeled as simple baseband linear system with three main parameters, the Photodiode (PD) current $\dot{Y}(t)$, optical input power $\dot{X}(t)$, and impulse response $h(t)$. Outdoor VLC applications are highly sensitive to the direct expose of PD to any high power visible source, which in turn generates high intensity ambient induced shot noise in the PD.

Minimizing the receiver background can be easily achieved by using optical band pass filter, which will filter out all the optical spectrum except the desired colour. Notwithstanding that, it still adds shot noise, which is usually the limiting noise source for any receiver. Independently from $\dot{X}(t)$, Gaussian distribution is the most accurate model for any high intensity shot noise in the PD. On the other hand, the pre-amplifier noise at the receiver side has also Gaussian distribution and is independent from the optical signal [13]. Consequently, the VLC channel can be modeled as an Additive White Gaussian noise (AWGN) channel,

$$
\dot{Y}(t)=\gamma \dot{X}(t) \otimes h(t)+\hat{N}(t) .
$$

Here, the PD current $\dot{Y}(t)$ is a result of the convolution between the optical power and impulse response, $\gamma$ represents the detector responsivity and $\hat{N}(t)$ is the AWGN. Optical channel DC gain $H(0)$ can be determined by following the same analysis for LED Lambertian emission [13] and considering the geometry in Fig 1.

$$
H(0)=\left\{\begin{array}{cc}
\frac{(m+1) A_{p h}}{2 \pi d^{2}} \cos ^{m}(\varphi) T_{s} g(\psi) \cos (\psi), & 0<\psi<\psi_{c} \\
0 & \text { elsewhere }
\end{array}\right.
$$

where $d$ is the separation distance and $\psi_{c}$ is the PD field of view (FOV) representing the maximum incidence angle. $A_{p h}$ is the physical area of the PD and $T_{s}$ is the filter transmission coefficient. For an ideal optical filter, such as the case of our simulation assumption, $T_{s}=1.0$ (see TABLE I), $m$ is lampertian emission order, which is as a key parameter specifying the directivity of the transmitter as shown in (3). The chosen half-power angle $\hat{\phi}$ has a remarkable influence on the coverage range and pattern shape of the lambertian light 
source. By choosing narrower $\hat{\phi}$, we can directly increase the service range.

$$
\mathbf{m}=-\frac{\ln 2}{\ln (\cos \hat{\phi})}
$$

By defining the internal refractive index $(n)$ of the optical lens used in the receiver side, the optical concentrator gain $g(\psi)$ in (2) can be calculated from the following formula:

$$
g(\psi)=\left\{\begin{array}{cc}
\frac{n^{2}}{\sin ^{2} \psi_{c}}, & 0<\psi<\psi_{c} \\
0, & \text { elsewhere }
\end{array}\right.
$$

Finally, the average received optical power $P_{r}$ is a result of the additive path-loss to the transmitted power in $\mathrm{dB}$ scale

$$
P_{r}=\dot{P}_{t}+H(0),
$$

which results in signal component at the receiver side

$$
S=\gamma^{2} P_{r}^{2}
$$

2) Noise Model: Discrete and extended background sources are the main two sources for the daylight noise. The first source is corresponding to the sun optical beam and it is considered as the main challenge in any optical receiver design, based on the fact that this noise may or may not be in the LOS of the receiver, and considering as small as 2 meters of inter-vehicle distance in a platoon, we assume that the receiver is not directly exposed to the discrete noise. The extended background source assumed to have isotropic behaviour and equally effect to the entire received spectrum since the source of this noise is the skylight [13], and can be detected in any optical receiver FOV.

Following the analysis in [17], most background sources are described by a Blackbody radiation model, in which the spectral irradiance is as follows:

$$
W\left(\lambda, T_{B}\right)=\frac{2 \pi b_{p} c^{2}}{\lambda^{2}}\left[\frac{1}{e^{b_{p} c / \lambda k T_{B}}-1}\right] .
$$

Here $b_{p}$ is the Plancks constant, $c$ is the speed of light, $\lambda$ is the desired wavelength, $k$ and $T_{B}$ are the Boltzmanns constant and the average temperature of the sun surface, respectively. Based on this analysis, the irradiance that falls within the spectral range of the receiver optical filter is

$$
E_{d e t}=\int_{\lambda_{1}}^{\lambda_{2}} W\left(\lambda, T_{B}\right) d \lambda .
$$

Therefore, the background $E_{\text {det }}$ noise power detected by the optical receiver physical area is given by:

$$
P_{b g}=E_{d e t} T_{s} A_{p h} n^{2} .
$$

Total noise variance $N$ is the sum of the both shot and thermal noise by combining both (11) and (12) which yields in

$$
N=\sigma_{\text {shot }}^{2}+\sigma_{\text {thermal }}^{2} \text {. }
$$

Shot noise represents the shot noise contributions from both LED vehicle rear light and the intense ambient light during the day time as the following;

$$
\sigma_{\text {shot }}^{2}=2 q \gamma\left(P_{r \text { Signal }}\right) B+2 q \gamma P_{b g} I_{2} B
$$

where $q$ is the electronic charge, $B$ is the equivalent noise rectangular transmitter pulse shape [18]. The background noise power $P_{b g}$ determined using (9) is a time variable reaches its peak at 02:00 pm [15].

Thermal noise is uniformly distributed across the frequency spectrum and can be given by:

$$
\sigma_{\text {thermal }}^{2}=\frac{8 \pi k T_{A}}{G} \eta A I_{2} B^{2}+\frac{16 \pi^{2} k T_{A} \Gamma}{g_{m}} \eta^{2} A_{p h}^{2} I_{3} B^{3},
$$

where $T_{A}$ is the environment temperature, $G$ is the openloop voltage gain, $\eta$ is the channel noise factor, $g_{m}$ is the transconductance and $I_{3}$ is the noise bandwidth factor for a full raised-cosine pulse shape [18]. All of the modeled parameters are tabulated in Table I. Lastly, we can define the receiver electrical Signal-to-Noise Ratio (SNR) by finding the power ratio between the signal in (6) and the total background noise in (10).

$$
S N R=\frac{S}{N}
$$

3) Modulation Model: BER performance is related to the both coding and the chosen modulation techniques. In this study and because of its excellent compromise between the peak power and the receiver bandwidth requirements, as well as the simplicity of implementation, we consider for the studied model a binary level modulation scheme consisting of two equally likely symbols On-Off-Keying $(O O K)$.

$$
B E R=Q(\sqrt{S N R})
$$

\section{B. Platoon model and control}

In this section, we first present the the vehicles kinematic model that govern the motion of a vehicle and which is used in our simulations, afterwards, we present a longitudinal controller based on a proportional integral (PI) controller and the proposed lateral controller based on a constant curvature approach.

1) Kinematic model: For simplicity and as depicted in Fig. 2(a), we approximate the kinematics of Ackerman steering mechanism of the vehicle to be a bicycle model, which has the same instantaneous centre of rotation (ICR), where $\delta, l$ and $\theta$ are respectively the steer angle, wheelbase and the orientation. In an inertial frame $F x y$, a vehicle position is defined by a position $(X, Y)$ and an orientation $\theta$. The kinematic model which governs the vehicles motion is given by:

$$
\left\{\begin{array}{l}
\dot{X}=V \cdot \cos (\theta) \\
\dot{Y}=V \cdot \sin (\theta) \\
\dot{\theta}=V \cdot \frac{\tan (\delta)}{l}
\end{array}\right.
$$

Fig. 2(b) shows a plan view for platooned two vehicle moving on a horizontal plan. These two vehicles have a longitudinal distance $\Delta x$, a lateral distance $\Delta y$ and an intervehicle distance $d$. In this study, we are interested in testing the basic tracking scenario of a platooned vehicles using VLC, where the main objective of the controllers is to follow the trajectory of the leader vehicle and maintaining a constant inter-vehicle distance. 


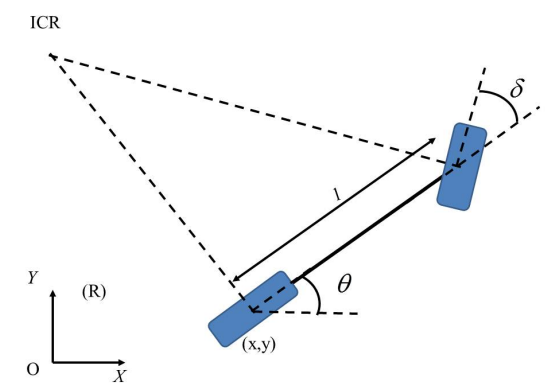

(a) Bicycle kinematic model approximate the vehicle model with the same instantaneous centre of rotation (ICR).

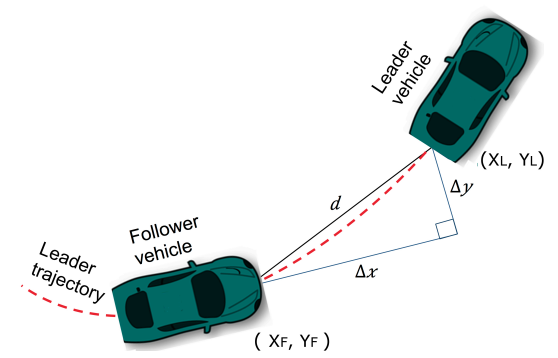

(b) Plan view for a platoon of two vehicles shows the longitudinal $(\Delta x)$ and lateral $(\Delta y)$ distances.

Fig. 2. Two vehicles (Leader and Follower) platoon model

2) Longitudinal and lateral control: In order to maintain a constant inter-distance reference $d_{\text {ref }}$, we use a classical proportional integral controller to control the velocity $V_{F}(t)$ at time $t$ of the follower vehicle. For a measured interdistance $d(t)$ at time $t$, the velocity of the follower vehicle is given by the following formula:

$$
V_{F}(t)=K_{p} \cdot e(t)+K_{i} \cdot \int_{0}^{t} e(\tau) \mathrm{d} \tau,
$$

where the error $e(t)$ is given by:

$$
e(t)=d_{r e f}-d(t) .
$$

We proposed a lateral controller based on a constant curvature approach. This controller allow the follower vehicle movement from its initial position $\left(X_{F}, Y_{F}\right)$ to the leader vehicle's position $\left(X_{L}, Y_{L}\right)$ with a constant steer angle $\delta$ as shown in Fig. 3. The steer angle $\delta$ is given by :

$$
\delta=\operatorname{atan}\left(\frac{2 \cdot l \cdot \sin (\phi)}{\sqrt{\Delta x^{2}+\Delta y^{2}}}\right)
$$

where

$$
\left\{\begin{array}{l}
\phi=\operatorname{atan}\left(\frac{\Delta y}{\Delta x}\right)-\theta \\
\Delta x=X_{L}-X_{F} \\
\Delta y=Y_{L}-Y_{F}
\end{array} .\right.
$$

\section{Proof}

Having a constant steer angle $\delta$, the vehicle's trajectory is a circle with a radius $R$ such as

$$
l=R \cdot \tan (\delta)
$$

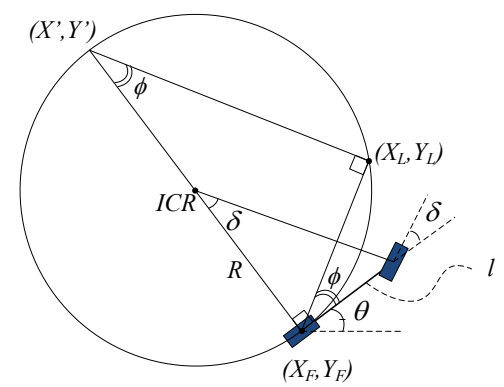

Fig. 3. Circle trajectory going from $\left(X_{F}, Y_{F}\right)$ to $\left(X_{L}, Y_{L}\right)$ with a constant steer angle $\delta$

and since the both positions $\left(X_{F}, Y_{F}\right)$ and $\left(X_{L}, Y_{L}\right)$ must be on the circle depicted in Fig. 3, the triangle defined by the three points $\left(X_{F}, Y_{F}\right),\left(X_{L}, Y_{L}\right)$ and $\left(X^{\prime}, Y^{\prime}\right)$ is square, thus

$$
\sqrt{\Delta x^{2}+\Delta y^{2}}=2 \cdot R \cdot \sin (\phi) .
$$

From (20) and (21) we can obtain the steer angle given by (18). Also from Fig. 3 we conclude that

$$
\tan (\phi+\theta)=\frac{\Delta y}{\Delta x}
$$

which proof and lead us back to the formula represented in (19).

\section{Performance Evaluation}

In this section, BER simulation results of the VLC channel are presented along with simulation results obtained from the Simulink model which employing the VLC characteristics for the platooned vehicles.

\section{A. Optical Channel Performance}

We determine the system performance through the $B E R$ plot for different incidence angle, electrical bandwidth and day time noise in order to determine the feasibility of platooning control using VLC. For numerical illustration, we assume the LED rear light with a dimension of $0.1 \mathrm{~m}^{2}$, where both receiver and transmitter have the same hight, with no vertical inclination angle and aligned with incidence and irradiance angles as illustrated in Fig. 1. The properties of the simulated transmitter and receiver are tabulated in Table I.

We consider the $O O K$ modulation scheme, $13.6 \mathrm{~dB}$ of SNR which is equivalent to $B E R=10^{-6}$, as the performance requirement for stable communication link; any configuration may result in $B E R>10^{-6}$ will be considered as connection failure. Fig. 4 compares the $B E R$ of different separation distance $d$ for the three main parameters of the studied model, Incidence Angle $\varphi$, Bandwidth $B$, and Ambient Noise Power $P_{b g}$, respectively. Our simulation results in Fig. 4.a indicate that 11 meters improvement of the separation distance between platooned vehicles can be achieved when the incidence angle of the transmitter decreases from $60^{\circ}$ to $20^{\circ}$, considering the worst ambient noise case $P_{b g}$ at 02:00 PM and for $B=10 \mathrm{MHz}$, which explains the strong influence of $\varphi$ in (2). The major drawback of any optical 
TABLE I

Simulation Model Parameters

\begin{tabular}{|l|l|}
\hline Parameter & Value \\
\hline \hline Filter Transmission coefficient, $T_{s}$ & 1.0 \\
Incidence/Irradiance Angle, $\varphi, \psi$ & $40^{\circ}$ \\
Photo Diode Responitivity, $\gamma$ & 0.56 \\
Semi Angle at Half Power, $\hat{\phi}$ & $60^{\circ}$ \\
Open Loop voltage Gain, $G$ & 10 \\
Noise Bandwidth Factor, $I_{2}$ & $10 \mathrm{MHz}$ \\
Receiver Field of View, $\psi_{c}$ & $60^{\circ}$ \\
Detector Physical Area, $A_{p h}$ & $1 \mathrm{~cm}^{2}$ \\
Output Optical Power, $P_{t}$ & $170 \mathrm{mw}$ \\
Electrical Bandwidth, $B$ & $10 \mathrm{MHz}$ \\
FET Transconductence, $g_{m}$ & $30 \mathrm{~ms}$ \\
Ambient Noise Power, $P_{b g}$ & $0.012 \mathrm{mw}$ \\
Refractive Index, $n$ & 1.5 \\
Wavelength, $\lambda$ & Red \\
\hline
\end{tabular}

receiver is the relatively wide optical bandwidth, which allows the receiver to collect large amount of background noise, that increases the ambient induced shot noise. Fig. 4.b illustrate the compromise between the optical coverage range and the electrical bandwidth of the receiver. For $\varphi=40^{\circ}$ and at background noise as in Fig. 4.a, the separation distance can reach up to 13 meters instead of only 4 meters if the chosen $B$ reduced from $100 \mathrm{Mhz}$ to $1 \mathrm{MHz}$.

Since all the simulation scenarios involving the SPCTRAL2 ambient noise model [15], we can predict the background noise power collected by the receiver with relatively high accuracy and for any day time, excluding the sun direct expose scenario. From Fig. 4.c, where incidence angle is fixed to be $40^{\circ}$ and $B=10 \mathrm{Mhz}$, it is interesting to remark that the separation distance can not exceed 7 meters if the vehicles are communicating during the ambient noise peak time $2: 00 \mathrm{pm}$, and this is due to the impact of the generated noise current as depicted in (11).

Fig. 4.d illustrates the optimal chosen values of the three main parameters in this study, $P_{b g}, B$ and $\varphi$ in order to achieve up to 7 meters inter-vehicle distance maintaining $B E R \leqslant 10^{-6}$. The chosen values support $\Delta y$ up to 5.36 meters and covering the average roads lane width. Finally, it should be noted that our calculation is made for $0.1 \mathrm{~m}^{2}$ PD physical area and single high intense LED, the communication distance can be largely increased by increasing the number of the LED's or the optical receiver physical aperture.

\section{B. Platooning Control using VLC}

We simulate a platoon of four vehicles with an initial inter-vehicle distance of 2 meters which falls in the range of the stable communication link offered by VLC model. We assume here that each vehicle knows its position, and we consider that the preceding vehicle will relay the required information to the next one in the queue using VLC communication, thus received information is processed to calculate the longitudinal and lateral distances and compute the required longitudinal and lateral control to maintain a
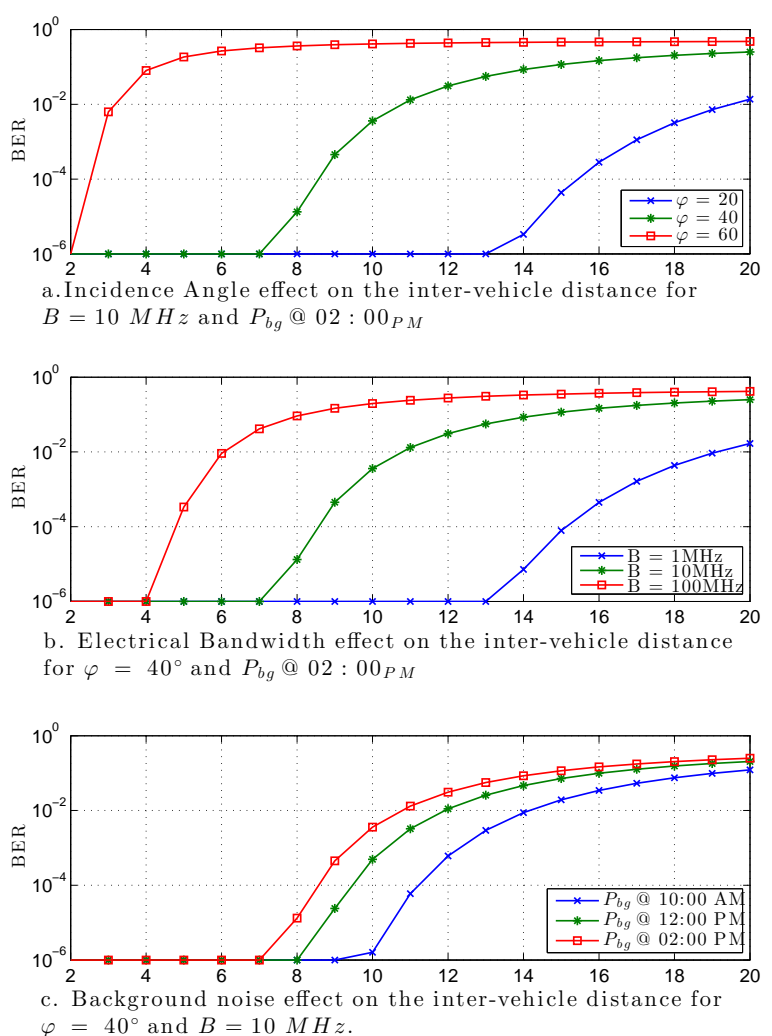

$\varphi=40^{\circ}$ and $B=10 \mathrm{MH}$

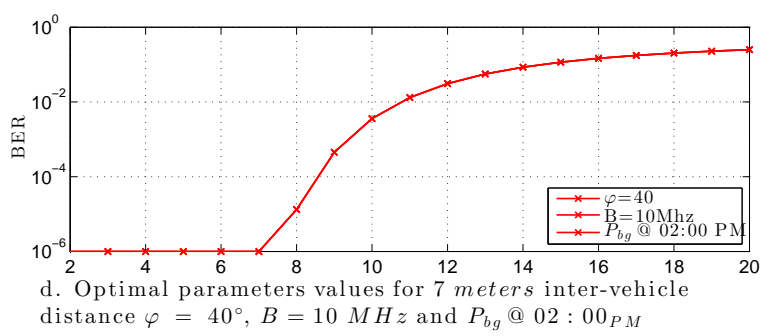

Fig. 4. Performance of the optical wireless channel for the three main parameters as a function of $B E R$ and the platoon inter-vehicle distance. a. Incidence Angle $(\varphi)$ effect, b. Electrical Bandwidth limitation. c.Ambient Noise Power $P_{b g}$ influence for different day time. d. represent the optimal parameter values to achieve stable VLC communication for inter-vehicle distance up to 7 meters.

constant inter-vehicle distance of 2 meters. The longitudinal and lateral controllers require local link in the sense that every follower vehicle should receive information from the one ahead. The controller is wholly dependent on the local links. To illustrate this, two cases were built in SIMULINK. Simulations show that the proposed lateral controller have a good performance with less processing time compared to other more sophisticated controllers. The maximum lateral error, which is the maximum spacing between the trajectory of the head vehicle and the tail vehicle, found to be less then $20 \mathrm{~cm}$. To illustrate this, two scenarios were built in in SIMULINK. The first scenario illustrated by Trajectory 1 in Fig.5, in this scenario, the curvature angle chosen to be less than $\psi_{c}$, allowing all the queued vehicles to preserve the requirements of the optical hardware, especially the FOV 


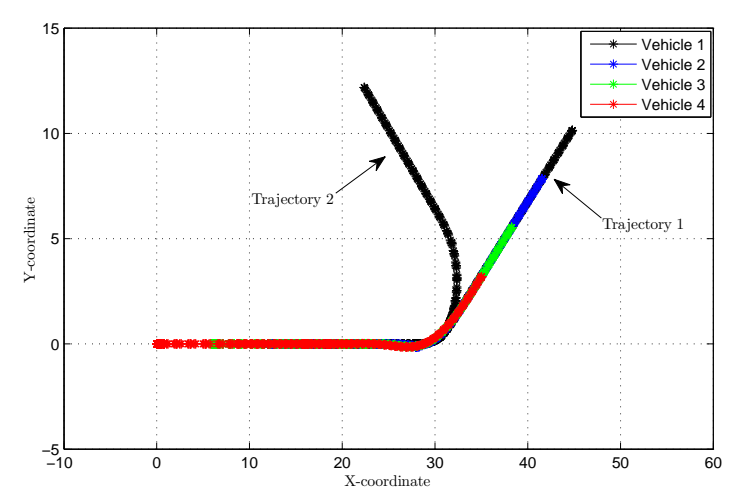

Fig. 5. Four vehicles platoon model in SIMULNK for two trajectories. Trajectory 1 illustrate the success scenario when the queued vehicle fall in the FOV of each other. Trajectory 2 illustrate the failure scenario when the trajectory curvature will cause out of FOV.

and and $B E R$ strict requirement. The second scenario (Fig. 5 trajectory 2) taking into account the communication link limits, and is expressed by sending stop (communication failure) signal to any vehicle located just after the link disconnection. for trajectory curvature angle larger than $\psi_{c}$, the vehicles 2, 3 and 4 have been stopped after losing the connection between the second vehicle and the leading one, which is the case when the decision is made that $B E R$ is higher than $10^{-6}$ due to the sharp curvature and being out of the FOV. The simulation results show the direct impact of the FOV and prove that as long as the road curvature exceeds the FOV, which is quite large angle, VLC can efficiently support platooning

\section{CONCLUSION}

In this paper, we have investigated analytical model; suggesting to employ the vehicles commercial rear lights as a reliable communication link. Furthermore, the main parameters that have significant effects on the outdoor VLC system performance and suiting platooning application were defined. The variation of these parameters such as incidence angle, receiver electrical bandwidth and day time noise were investigated and compared for different intervehicle distances. The simulation of the studied model shows that a $B E R$ of $10^{-6}$ which is equivalent to $S N R$ around $14.6 \mathrm{~dB}$ is achievable for separation distance up to 7 meters between the platooned vehicles. We also implemented a Matlab/SIMULINK based platoon model with a longitudinal and a lateral controllers for each vehicle considering the VLC communication model limitations. We improved simulations for two scenarios in order to point out the problem of losing VLC communication in some curved trajectories.

For further research, the effects of acceleration limits, mobility and vehicles vibrations need to be studied because they are known to induce system instability. Since we find that the VLC links are very directional in transmission and reception by the strong influence of the incidence and irradiation angles, we contemplate that beam steering techniques may also be applied to VLC. Also, we expect complementary corporation between VLC and RF solutions where both can be working together to support the diverse requirements of the vehicular applications, e.g., using VLC in dense traffic conditions while switching to RF for long range or sharp road curvatures. Finally, applying the results of this research to real platoon demonstration needs to be addressed and the method of selecting control and optical parameters warrants further in-depth investigation.

\section{REFERENCES}

[1] W. Dunbar and D. Caveney, "Distributed receding horizon control of vehicle platoons: Stability and string stability," Automatic Control, IEEE Transactions on, vol. 57, no. 3, pp. 620-633, March 2012.

[2] S. Eichler, "Performance evaluation of the IEEE 802.11p WAVE communication standard," in Vehicular Technology Conference, 2007. VTC-2007 Fall. 2007 IEEE 66th, 2007, pp. 2199-2203.

[3] K. Bilstrup, E. Uhlemann, E. Strm, and U. Bilstrup, "On the ability of the 802.11p MAC method and STDMA to support real-time vehicle-to-vehicle communication," vol. 2009, no. 1, p. 902414, Jan. 2009. [Online]. Available: http://jwcn.eurasipjournals.com/content/ 2009/1/902414/abstract

[4] M. Khabazian, S. Aissa, and M. Mehmet-Ali, "Performance modeling of message dissemination in vehicular ad hoc networks," in 2010 5th IEEE International Symposium on Wireless Pervasive Computing (ISWPC), 2010, pp. 174-179.

[5] "IEEE standard for local and metropolitan area NetworksPart 15.7: Short-range wireless optical communication using visible light," pp. $1-309,2011$.

[6] F. Deicke, W. Fisher, and M. Faulwasser, "Optical wireless communication to eco-system," in Future Network Mobile Summit (FutureNetw), 2012, 2012, pp. 1-8.

[7] A. Cailean, B. Cagneau, L. Chassagne, S. Topsu, Y. Alayli, and J.-M. Blosseville, "Visible light communications: Application to cooperation between vehicles and road infrastructures," in 2012 IEEE Intelligent Vehicles Symposium (IV), 2012, pp. 1055-1059.

[8] S. Shladover, "Path at 20 - history and major milestones," in Intelligent Transportation Systems Conference, 2006. ITSC '06. IEEE, 2006, pp. 122-129.

[9] P. Daviet and M. Parent, "Longitudinal and lateral servoing of vehicles in a platoon," in Intelligent Vehicles Symposium, 1996., Proceedings of the 1996 IEEE, 1996, pp. 41-46.

[10] S. Hall, B. Chaib-draa, and J. Laumonier, "Car platoons simulated as a multiagent system," in In: Proc. 4th Workshop on Agent-Based Simulation, 2003, pp. 57-63.

[11] E. C. Tom Robinson, Eric Chan, "Operating platoons on public motorways: An introduction to the sartre platooning programme," in 17th World Congress on Intelligent Transport Systems (ITS) 2010, October 2010.

[12] P. Fernandes and U. Nunes, "Platooning with dsrc-based ivc-enabled autonomous vehicles: Adding infrared communications for ivc reliability improvement," in Intelligent Vehicles Symposium (IV), 2012 IEEE, 2012, pp. 517-522.

[13] J. Kahn and J. Barry, "Wireless infrared communications," vol. 85, no. 2, pp. $265-298$, Feb. 1997.

[14] C. B. Liu, B. Sadeghi, and E. W. Knightly, "Enabling vehicular visible light communication (V2LC) networks," in Proceedings of the Eighth ACM international workshop on Vehicular inter-networking, ser. VANET '11. New York, NY, USA: ACM, 2011, p. 4150. [Online]. Available: http://doi.acm.org/10.1145/2030698.2030705

[15] I. Lee, M. Sim, and F. Kung, "Performance enhancement of outdoor visible-light communication system using selective combining receiver," vol. 3, no. 1, pp. $30-39$, Feb. 2009.

[16] S. Okada, T. Yendo, T. Yamazato, T. Fujii, M. Tanimoto, and Y. Kimura, "On-vehicle receiver for distant visible light road-tovehicle communication," in 2009 IEEE Intelligent Vehicles Symposium, 2009, pp. 1033-1038.

[17] R. E. Bird and C. Riordan, "Simple solar spectral model for direct and diffuse irradiance on horizontal and tilted planes at the earth's surface for cloudless atmospheres," vol. 25, no. 1, pp. 87-97, Jan. 1986. [Online]. Available: http://journals.ametsoc.org/doi/abs/10. 1175/1520-0450(1986)025\%3C0087\%3ASSSMFD\%3E2.0.CO\%3B2

[18] J. R. Barry, Wireless Infrared Communications. Springer, 1994. 